\title{
The construction of simplicial groups in digital images
}

\author{
Simge Öztunç ${ }^{*}$, Necdet Bildik and Ali Mutlu
}

"Correspondence:

simge.oztunc@cbu.edu.tr

Mathematics Department, Faculty

of Science and Arts, Celal Bayar

University, Manisa, 45047, Turkey

\begin{abstract}
In this paper, we recall some definitions and properties from digital topology, and we consider the simplices and simplicial complexes in digital images due to adjacency relations. Then we define the simplicial set and conclude that the simplicial identities are satisfied in digital images. Finally, we construct the group structure in digital images and define the simplicial groups in digital images. Consequently, we calculate the digital homology group of two-dimensional digital simplicial group.
\end{abstract}

MSC: 55N35; 68R10; 68U10; 18G30

Keywords: digital image; adjacency relation; simplicial map; face and degenerate map; simplicial set; simplicial group

\section{Introduction and basic concepts}

Digital topology is a branch of mathematics where the image processing and digital image processing are studied. Many mathematicians such as Rosenfeld [1], Han [2], Kong [3, 4], Malgouyres [5], Boxer [6-8], Karaca [9-11] and others have contributed to this area with their research. The notions of a digital image, a digital continuous map and digital homotopy were studied in literature $[1,4,5,7,8]$. Their recognition and efficient computation became a useful material for our study.

On the other hand, simplicial groups were first studied by Kan [12] in the 1950s. We carry the notion of a digital image on to the simplicial groups and construct algebraic structures in digital images such as simplicial sets, simplicial groups by using the figures and definitions in [13]. In dimension one, we use the 2-adjacency relation, and in dimension two, we use the 8-adjacency relation.

Assume that $\mathbb{Z}^{n}$ is $n$-dimensional Euclidean spaces. A finite subset of $\mathbb{Z}^{n}$ with an adjacency relation is called to be a digital image.

Definition 1.1 ([4]; see also [14] and [9])

(1) Two points $p$ and $q$ in $\mathbb{Z}$ are 2-adjacent if $|p-q|=1$.

(2) Two points $p$ and $q$ in $\mathbb{Z}^{2}$ are 8 -adjacent if they are distinct and differ by at most 1 in each coordinate.

(3) Two points $p$ and $q$ in $\mathbb{Z}^{2}$ are 4-adjacent if they are 8-adjacent and differ by exactly one coordinate.

(4) Two points $p$ and $q$ in $\mathbb{Z}^{3}$ are 26-adjacent if they are distinct and differ by at most 1 in each coordinate.

(c) 2013 Öztunç et al.; licensee Springer. This is an Open Access article distributed under the terms of the Creative Commons Attribution License (http://creativecommons.org/licenses/by/2.0), which permits unrestricted use, distribution, and reproduction in any medium, provided the original work is properly cited. 

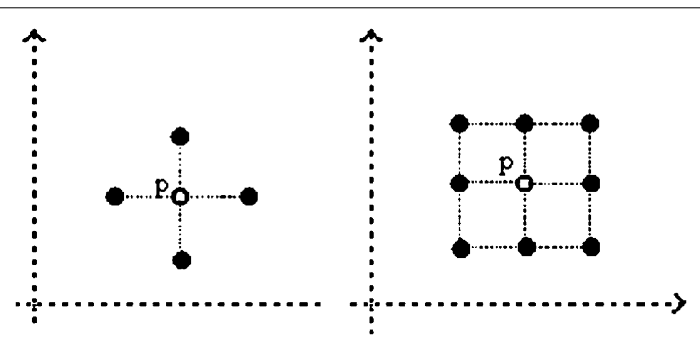

Figure 2 4-adjacency, 8-adjacency.
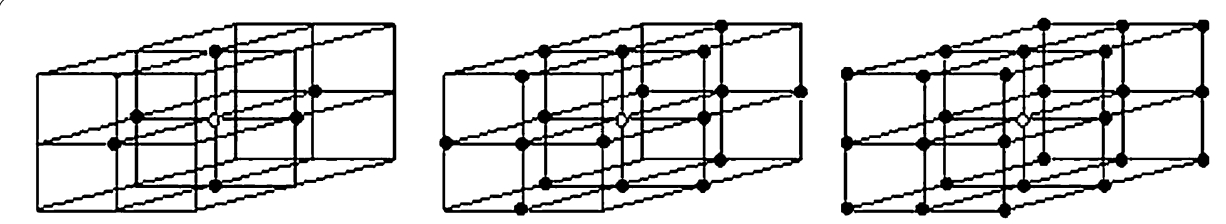

Figure 3 6-adjacency, 18-adjacency, 26-adjacency.

(5) Two points $p$ and $q$ in $\mathbb{Z}^{3}$ are 18-adjacent if they are 26-adjacent and differ in at most two coordinates.

(6) Two points $p$ and $q$ in $\mathbb{Z}^{3}$ are 6-adjacent if they are 18-adjacent and differ by exactly one coordinate (see Figures 1-3).

Suppose that $\kappa$ is an adjacency relation defined on $\mathbb{Z}^{n}$. A digital image $X \subset \mathbb{Z}^{n}$ is $\kappa$ connected [15] if and only if for every pair of points $\{x, y\} \subset X, x \neq y$, there is a set $\left\{x_{0}, x_{1}, \ldots, x_{c}\right\} \subset X$ such that $x=x_{0}, x_{c}=y$ and $x_{i}$ and $x_{i+1}$ are $\kappa$-neighbors, $i \in\{0,1, \ldots$, $c-1\}$.

Definition 1.2 Let $X$ and $Y$ be digital images such that $X \subset \mathbb{Z}^{n_{0}}, Y \subset \mathbb{Z}^{n_{1}}$. Then the digital function $f: X \rightarrow Y$ is a function which is defined between digital images.

Definition 1.3 ([6]; see also [7] and [8]) Let $X$ and $Y$ be digital images such that $X \subset \mathbb{Z}^{n_{0}}$, $Y \subset \mathbb{Z}^{n_{1}}$. Assume that $f: X \rightarrow Y$ is a function. Let $\kappa_{i}$ be an adjacency relation defined on $\mathbb{Z}^{n_{i}}, i \in\{0,1\}$. Then $f$ is called to be $\left(\kappa_{0}, \kappa_{1}\right)$-continuous if the image under $f$ of every $\kappa_{0}$-connected subset of $X$ is $\kappa_{1}$-connected.

A function which is defined in Definition 1.3 is referred to be digitally continuous. A consequence of this definition is given below.

Definition 1.4 ([6]; see also [8]) Let $X$ and $Y$ be digital images. Then the function $f: X \rightarrow$ $Y$ is said to be $\left(\kappa_{0}, \kappa_{1}\right)$-continuous if and only if for every $\{x, y\} \subset X$ such that $x_{0}$ and $x_{1}$ are $\kappa_{0}$-adjacent, either $f\left(x_{0}\right)=f\left(x_{1}\right)$ or $f\left(x_{0}\right)$ and $f\left(x_{1}\right)$ are $\kappa_{1}$-adjacent. 
Definition 1.5 ([6]) Let $a, b \in \mathbb{Z}, a<b$. A digital interval is a set of the form

$$
[a, b]_{\mathbb{Z}}=\{z \in \mathbb{Z} \mid a \leq z \leq b\}
$$

in which 2-adjacency is assumed.

For instance, if $\kappa$ is an adjacency relation on a digital image $Y$, then $f:[a, b]_{\mathbb{Z}} \rightarrow Y$ is $(2, \kappa)$-connected if and only if for every $\{c, c+1\} \subset[a, b]_{\mathbb{Z}}$, either $f(c)=f(c+1)$ or $f(c)$ and $f(c+1)$ are $\kappa$-adjacent.

Definition 1.6 ([14]) Let $S$ be a set of nonempty subsets of a digital image $(X, \kappa)$. Then the members of $S$ are called simplices of $(X, \kappa)$ if the following hold:

(a) If $p$ and $q$ are distinct points of $s \in S$, then $p$ and $q$ are $\kappa$-adjacent.

(b) If $s \in S$ and $\emptyset \neq t \subset s$, then $t \in S$ (note that this implies that every point $p$ belonging to a simplex determines a simplex $\{p\})$.

An $m$-simplex is a simplex $S$ such that $|S|=m+1$.

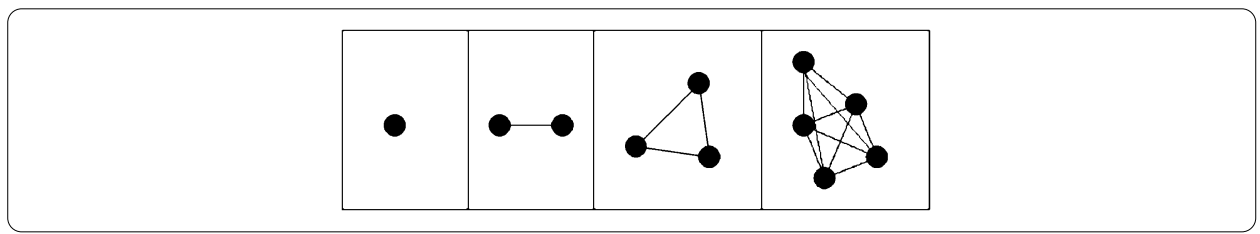

Definition $1.7([9,14])$ Let $(X, \kappa)$ be a finite collection of digital $m$-simplices, $0 \leq m \leq d$, for some nonnegative integer $d$. If the following statements hold, then $(X, \kappa)$ is called a finite digital simplicial complex:

(1) If $P$ belongs to $X$, then every face of $P$ also belongs to $X$.

(2) If $P, Q \in X$, then $P \cap Q$ is either empty or a common face of $P$ and $Q$.

The dimension of a digital simplicial complex $X$ is the largest integer $m$ such that $X$ has an $m$-simplex.

Example 1.8 If $X$ is a digital simplicial complex and $\left[v_{i_{0}}, \ldots, v_{i_{m}}\right]$ is a $(\kappa, m)$-simplex of $X$, then any subset of $\left\{v_{i_{0}}, \ldots, v_{i_{m}}\right\} \subset\left(\mathbb{Z}^{n}, \kappa\right)$ is a face of that digital simplex and thus is itself a digital simplex of $X$. In particular, we can think of the $(\kappa, m)$-simplex $\left[v_{i_{0}}, \ldots, v_{i_{m}}\right]$ as a geometric digital simplicial complex consisting of itself and its face.

\section{Digital simplicial maps}

If $K$ and $L$ are geometric digital simplicial complexes, then a digital simplicial map $f$ : $K \rightarrow L$ is determined by taking the vertices $\left\{v_{i}\right\}$ of $K$ to vertices $\left\{f\left(v_{i}\right)\right\}$ of $L$ such that if $\left[v_{i_{0}}, \ldots, v_{i_{m}}\right]$ is a $\left(\kappa_{1}, m\right)$-simplex of $K$, then $f\left(v_{i_{0}}\right), \ldots, f\left(v_{i_{m}}\right)$ are all vertices (not necessarily unique) of some $\left(\kappa_{2}, m\right)$-simplex in $L$. Given such a function $K^{0} \rightarrow L^{0}$, the rest of $f: K \rightarrow L$ is determined by linear interpolation on each $\left(\kappa_{1}, m\right)$-simplex (if $x \in K$ can be represented by $x=\sum_{j=1}^{m} t_{j} v_{i_{j}}$ in barycentric coordinates of the $\left(\kappa_{1}, m\right)$-simplex spanned by the $v_{i_{j}}$, then $\left.f(x)=\sum_{j=1}^{m} t_{j} f\left(v_{i_{j}}\right)\right)$ (see Figures 4,5). 


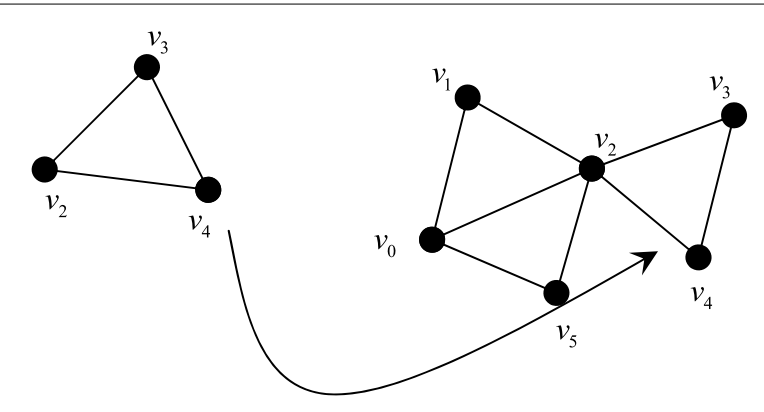

Figure 4 Including the simplex $\left[v_{2}, v_{3}, v_{4}\right]$ into a larger digital simplicial complex.

Figure 5 A collapse of a $(8,2)$-simplex to a $(2,1)$-simplex.

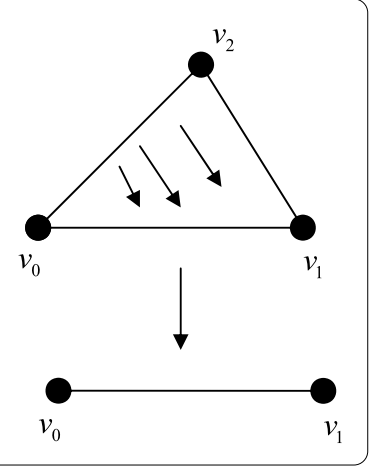

Corollary 2.1 Every digital simplicial map is digitally continuous.

Proof Let $K$ and $L$ be digital simplicial complexes having $\kappa_{1}$ and $\kappa_{2}$ adjacency, respectively, and let $f: K \rightarrow L$ be a digital simplicial map. Since $K$ is a simplicial complex, then the point of simplices of $K$ have $\kappa_{1}$ adjacency. So, $f$ maps the points of the simplices of $K$ to the points of the simplices of $L$. Therefore the image of $\kappa_{1}$-adjacent points under $f$ is $\kappa_{2}$-adjacent points. Consequently, $f$ is $\left(\kappa_{1}, \kappa_{2}\right)$-continuous, i.e., digitally continuous.

Example 2.2 A simple but interesting and important example is the inclusion of a $(\kappa, m)$ simplex into a digital simplicial complex. If $X$ is a digital simplicial complex and $v_{i_{0}}, \ldots, v_{i_{m}}$ is a collection of vertices of $X$ that spans a $(\kappa, m)$-simplex of $X$, then $K=\left[v_{i_{0}}, \ldots, v_{i_{m}}\right]$ is itself a digital simplicial complex. We then have a simplicial map $K \rightarrow X$ that takes each $v_{i_{j}}$ to the corresponding vertex in $X$ and hence takes $K$ identically to itself inside $X$.

Example 2.3 Let $\left[v_{0}, v_{1}, v_{2}\right]$ be a $(8,2)$-simplex, one of whose $(8,1)$-faces is $\left[v_{0}, v_{1}\right]$. Consider the simplicial map $f:\left[v_{0}, v_{1}, v_{2}\right] \rightarrow\left[v_{0}, v_{1}\right]$, determined by $f\left(v_{0}\right)=v_{0}, f\left(v_{1}\right)=v_{1}$, $f\left(v_{2}\right)=v_{1}$, that collapses the $(8,2)$-simplex down to the $(8,1)$-simplex (see Figure 5$)$. The great benefit of the theory of digital simplicial sets is a way to generalize these kinds of maps in order to preserve information so that we can still see the image of the $(\kappa, 2)$ simplex hiding in the $(\kappa, 1)$-simplex as a degenerate $(\kappa, m)$-simplex.

\subsection{Face maps in digital images}

Given a $(\kappa, n)$-simplex, we would like a handy way of referring to its $(n-1)$-dimensional faces. This is handled by the face maps. On the standard $(\kappa, n)$-simplex, we have $n+1$ face 


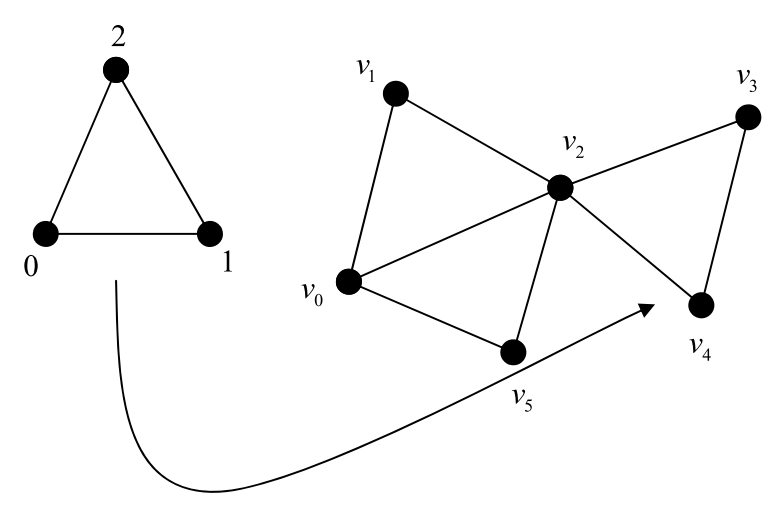

Figure $6\left[v_{2}, v_{3}, v_{4}\right]$ as image of $\left|\Delta^{2}\right|_{\kappa}$.

Figure 7 The face maps of $\left|\Delta^{2}\right|_{8}$. Note that the arrows denote assignments and also not continuous maps of spaces.

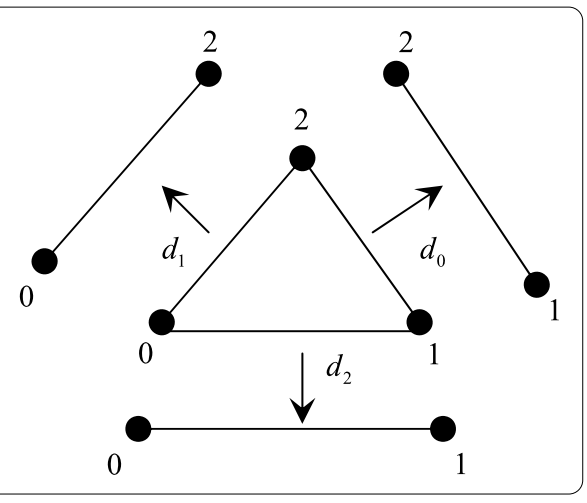

maps $d_{0}, \ldots, d_{n}$, defined so that $d_{j}[0, \ldots, n]=[0, \ldots, \widehat{j}, \ldots, n]$, where, as usual, the $\widehat{-}$ denotes a term that is being omitted. Thus applying $d_{j}$ to $[0, \ldots, n]$ yields the $(n-1)$-face missing the vertex $j$. It is important to note that each $d_{j}$ simply assigns one of its faces to the $(\kappa, n)$ simplex (see Figures 6, 7).

\subsection{Delta sets and delta maps in digital images}

Delta sets (sometimes called $\Delta_{\kappa}$-sets) constitute an intermediary between digital simplicial complexes and digital simplicial sets.

Definition 2.4 A delta set consists of a sequence of sets $X_{0}, X_{1}, \ldots$ which have $\kappa_{0}, \kappa_{1}, \ldots$ adjacency respectively, and the maps $d_{i}: X_{n+1} \rightarrow X_{n}$ for each $i$ and $n \geq 0,0 \leq i \leq n+1$, such that $d_{i} d_{j}=d_{j-1} d_{i}$ whenever $i<j$.

Of course this is just an abstraction, and generalization, of the definition of an ordered digital simplicial complex, in which $X_{n}$ are the sets of $(\kappa, n)$-simplices and the $d_{i}$ are the digital face maps.

\subsection{Degenerate maps in digital images}

Degeneracy maps in digital images are, in some sense, the conceptual converse of face maps. Recall that the face map $d_{j}$ takes a $(\kappa, n)$-simplex and gives us back its $j$ th $(n-1)$ face. On the other hand, the $j$ th digital degeneracy map $s_{j}$ takes a $(\kappa, n)$-simplex and gives us back the $j$ th degenerate $(\kappa,(n+1))$-simplex in digital images living inside it. 
As usual, we illustrate with the standard $(\kappa, n)$-simplex, which will be a model for what happens in all digital simplicial sets. Given the standard $(\kappa, n)$-simplex as $\left|\Delta^{2}\right|_{\kappa}=[0, \ldots, n]$, there are $(n+1)$-digital degeneracy maps $s_{0}, \ldots, s_{n}$, defined by $s_{j}[0, \ldots, n]=[0, \ldots, j, j, \ldots, n]$. In other words, $s_{j}[0, \ldots, n]$ gives us the unique degenerate $(\kappa,(n+1))$-simplex in $\left|\Delta^{n}\right|_{\kappa}$ with only the $j$ th vertex repeated.

Again, the geometric concept is that $s_{j}\left|\Delta^{n}\right|_{\kappa}$ can be thought of as the process of collapsing $\Delta^{n+1}$ down into $\Delta^{n}$ by the digital simplicial map $\pi_{j}$ defined by $\pi_{j}(i)=i$ for $i<j, \pi_{j}(j)=$ $\pi_{j}(j+1)=j$ and $\pi_{j}(j)=i-1$ for $i>j+1$.

This idea extends naturally to digital simplicial complexes, to digital delta sets, and also to $(\kappa, n)-1$ simplices that are already degenerate. If we have a (possibly degenerate) $(\kappa, n)$-simplex $\left[v_{i_{0}}, \ldots, v_{i_{n}}\right]$ with $i_{k} \leq i_{k+1}$ for each $k, 0 \leq k<n$, then we set $s_{j}\left[v_{i_{0}}, \ldots, v_{i_{n}}\right]=$ $\left[v_{i_{0}}, \ldots, v_{i_{j}}, v_{i_{j}}, \ldots, v_{i_{n}}\right]$, i.e., $v_{i_{j}}$ always repeats. Thus we may say that this is a digital degenerate simplex in $\left[v_{i_{0}}, \ldots, v_{i_{n}}\right]$.

Also, as for the $d_{i}$, there are certain natural relations that the degeneracy maps possess. In particular, if $i \leq j$, then $s_{i} s_{j}[0, \ldots, n]=[0, \ldots, i, i, \ldots, j, j, \ldots, n]=s_{j+1} s_{i}[0, \ldots, n]$. Note that we have $s_{j+1}$ in the last formula, not $s_{j}$, since the application of $s_{i}$ pushes $j$ one slot to the right.

Furthermore, there are relations amongst the face and degeneracy operators. These are a little bit advantageous to write down since there are three possibilities:

$$
\begin{aligned}
& d_{i} s_{j}=s_{j-1} d_{i}, \quad i<j, \\
& d_{j} s_{j}=d_{j+1} s_{j}=i d, \\
& d_{i} s_{j}=s_{j} d_{i-1}, \quad i>j+1 .
\end{aligned}
$$

These situations are all clear. For example, applying $[0, \ldots, n]$ to either side of the first formula yields $[0, \ldots, \widehat{\imath}, \ldots, j, j, \ldots, n]$. Note also that the middle formula takes care of both $i=j$ and $i=j+1$.

\section{The structure of a simplicial set in digital images}

Now we give the definition of digital simplicial sets.

Definition 3.1 A digital simplicial set consists of a sequence of sets $X_{0}, X_{1}, \ldots$ which have $\kappa_{0}, \kappa_{1}, \ldots$ adjacency respectively, the functions $d_{i}: X_{n} \rightarrow X_{n-1}$ and $s_{i}: X_{n} \rightarrow X_{n+1}$ for each $i$ and $n \geq 0$ with $0 \leq i \leq n$ are such that

$$
\begin{aligned}
& d_{i} d_{j}=d_{j-1} d_{i}, \quad i<j, \\
& d_{i} s_{j}=s_{j-1} d_{i}, \quad i<j, \\
& d_{j} s_{j}=d_{j+1} s_{j}=i d, \\
& d_{i} s_{j}=s_{j} d_{i-1}, \quad i>j+1, \\
& s_{i} s_{j}=s_{j+1} s_{i}, \quad i \leq j .
\end{aligned}
$$

Example 3.2 If the standard $(2,0)$-simplex $X=[0]$ is now realized as a digital simplicial set, then it is the unique digital simplicial set with one element in each $X_{n}, n \geq 0$. Thus the element in dimension $n$ is $[\underbrace{0,0, \ldots, 0}_{n+1 \text {-times }}]$. 

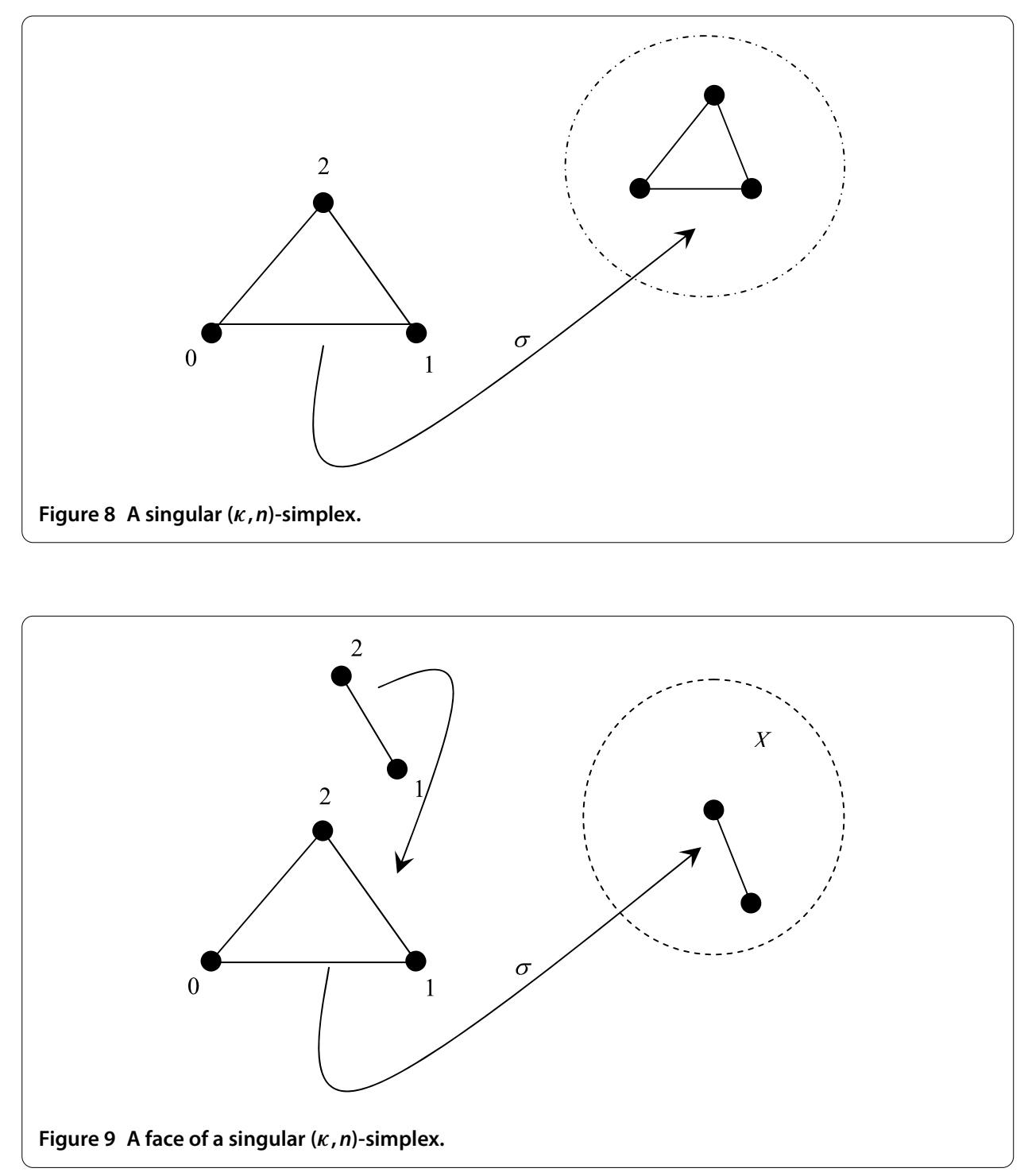

Example 3.3 As a digital simplicial set, the standard ordered $(2,1)$-simplex $X=[0,1]$ already has $n+2$ elements in each $X_{n}$. Namely, $X_{2}=\{[0,0,0],[0,0,1],[0,1,1],[1,1,1]\}$.

\subsection{Nondegenerate simplices in digital images}

$\mathrm{A}(\kappa, n)$-simplex $x \in X_{n}$ is called nondegenerate if $x$ cannot be written as $s_{i} y$ for any $y \in X_{n-1}$ and any $i$.

Every $(\kappa, n)$-simplex of a digital simplicial complex or digital delta set is a nondegenerate simplex of the corresponding digital simplicial set. If $Y$ is a topological space, then a $(\kappa, n)$-simplex of $S(Y)$ is nondegenerate. It cannot be written as the composition $\Delta_{\kappa}^{n} \stackrel{\pi}{\longrightarrow} \Delta_{\kappa}^{k} \stackrel{\sigma}{\longrightarrow} Y$, where $\pi$ is a simplicial collapse with $k<n$ and $\sigma$ is a singular $(\kappa, k)$ simplex (see Figures 8-10).

Note that it is possible for a nondegenerate $(\kappa, n)$-simplex to have a degenerate $(\kappa, n)$ face. It is also possible for a degenerate simplex to have a nondegenerate face (for example, we know $d_{j} s_{j} x=x$ for any $x$, it is degenerate or not). 


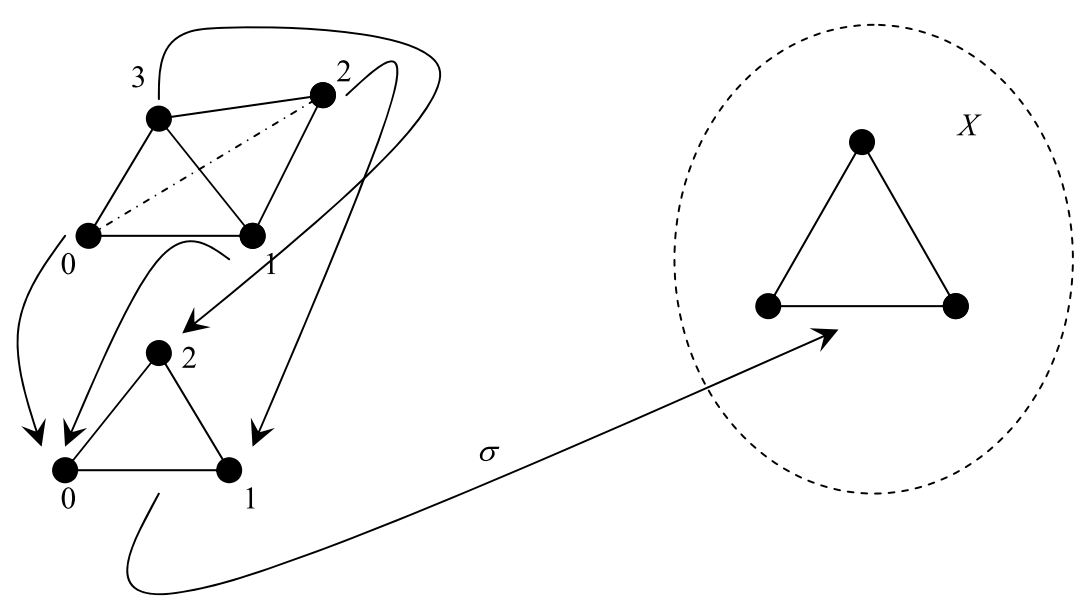

Figure 10 A degenerate $(\kappa, n)$-singular simplex.

\subsection{Categorical definition}

As for digital delta sets, the basic properties of simplicial sets derive from those of the standard ordered $(\kappa, n)$-simplex. In fact, that is where the prototypes of both the face and degeneracy maps live and where we first developed the axioms relating them. Thus it is not surprising (at this point) that there is a categorical definition of digital simplicial sets, analogous to the one for delta sets, in which each digital simplicial set is the functorial image of a category and $\Delta$ is built from the standard digital simplices.

Definition 3.4 The category $\Delta_{\kappa}$ has the finite ordered sets $[n]_{\kappa}=\{0,1,2, \ldots, n\}$ as objects. The morphisms of $\Delta_{\kappa}$ are order-preserving functions $[m]_{\kappa} \rightarrow[n]_{\kappa}$.

Definition 3.5 (Categorical definition of digital simplicial set) Let DSet be a category of digital sets, that is, DSet has digital images as objects and digital functions as morphisms. A digital simplicial set is a contravariant functor $X: \Delta_{\kappa} \rightarrow D S e t$ (equivalently, a covariant functor $X: \Delta_{\kappa}^{o p} \rightarrow D S e t$ ).

\section{Simplicial groups in digital images}

Group axioms are satisfied in a finite subset of $\mathbb{Z}^{n}$, including the $(0,0, \ldots, 0)$ point.

Definition 4.1 Let $\mathcal{G}$ be a subset of the digital image which has $\kappa$-adjacency relation. A simplicial group $\mathcal{G}$ in digital images consists of a sequence of groups $\mathcal{G}_{n}$ and collections of group homomorphisms $d_{i}: \mathcal{G}_{n} \rightarrow \mathcal{G}_{n-1}$ and $s_{i}: \mathcal{G}_{n} \rightarrow \mathcal{G}_{n+1}, 0 \leq i \leq n$, that satisfy the following axioms:

$$
\begin{aligned}
& d_{i} d_{j}=d_{j-1} d_{i}, \quad i<j, \\
& d_{i} s_{j}=s_{j-1} d_{i}, \quad i<j, \\
& d_{j} s_{j}=d_{j+1} s_{j}=i d, \\
& d_{i} s_{j}=s_{j} d_{i-1}, \quad i>j+1, \\
& s_{i} s_{j}=s_{j+1} s_{i}, \quad i \leq j .
\end{aligned}
$$


Figure 11 A pictoral representation of a $(8,2)$-simplex of a simplicial group with arrows representing the face morphisms from dimension 2 to dimension 1 and from dimension 1 to dimension 0 .

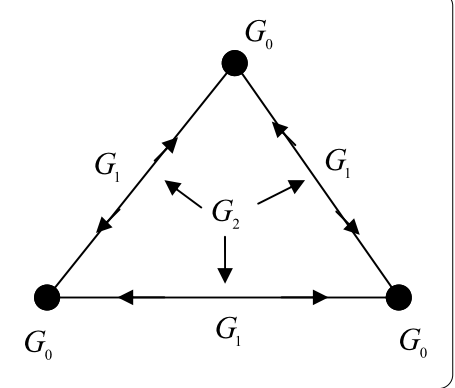

An example of a simplicial group in a digital image can be seen in Figure 11.

Example 4.2 Suppose $X$ is a digital simplicial set with $\kappa$-adjacency. Then we may define the simplicial group $C_{*}(X)$ having $\left(C_{*} X\right)_{n}=C_{n}(X)$ as a free abelian group generated by the elements of $X_{n}$ with $d_{i}$ and $s_{i}$ in $C_{*}(X)$ taken to be linear extensions of the face maps $d_{i}$ and $s_{i}$ of $X$.

We can also present the total face map $d=\sum_{i=0}^{n}(-1)^{i} d_{i}: C_{n}(X) \rightarrow C_{n-1}(X)$ and then define the homology $H_{*}(X)$ as the homology of the chain complex $\left(C_{*}(X), d\right)$.

Example 4.3 Let us give a remarkable example for a simplicial group in digital images, which is important in the theory of homology of groups in digital images. Assume that $G$ is a group of digital images and $D G$ is the simplicial group defined as follows. Let $D G_{n}=G^{\times n}$ be the product of $G$ with itself $n$ times. Thus $G^{\times 0}$ is just the trivial group $\{e\}$. For an element $\left(g_{1}, \ldots, g_{n}\right) \in D G_{n}$, let

$$
\begin{aligned}
& d_{0}\left(g_{1}, \ldots, g_{n}\right)=\left(g_{2}, \ldots, g_{n}\right), \\
& d_{i}\left(g_{1}, \ldots, g_{n}\right)=\left(g_{1}, \ldots, g_{i-1}, g_{i+1}, \ldots, g_{n}\right), \quad 0<i<n, \\
& d_{n}\left(g_{1}, \ldots, g_{n}\right)=\left(g_{1}, \ldots, g_{n-1}\right), \\
& s_{i}\left(g_{1}, \ldots, g_{n}\right)=\left(g_{1}, \ldots, g_{i}, e, g_{i+1}, g_{n}\right) .
\end{aligned}
$$

This defines a simplicial group in digital images. The realization of an underlying digital simplicial set turns out to be the classifying space of the group $G$, and so the homology $H_{*}(D G)$ coincides with group homology of the group $G$.

\section{Digital homology groups of simplicial groups}

In this section, we consider $X$ as a digital simplicial group, and we use the method of calculations given by [14] and [9].

Definition 5.1 $C_{n}(X)$ is a free abelian group with basis of all digital $(\kappa, n)$-simplices in $X$.

Corollary 5.2 Let $X$ be an $m$-dimensional digital simplicial group with $\kappa$-adjacency. Then if $q>m$, then $C_{q}^{\kappa}(X)$ is a trivial group. 
Definition 5.3 Let $(X, \kappa) \subset \mathbb{Z}^{n}$ be an $m$-dimensional digital simplicial group. The homomorphism $\partial_{q}: C_{q}^{\kappa}(X) \rightarrow C_{q-1}^{\kappa}(X)$ defined by

$$
\partial_{q}\left(\left\langle x_{0}, \ldots, x_{q}\right\rangle\right)= \begin{cases}\sum_{i=0}^{q}(-1)^{i}\left\langle p_{0}, \ldots, \hat{p}_{i}, \ldots, p_{q}\right\rangle, & q \leq m, \\ 0, & q>m\end{cases}
$$

is called boundary homomorphism. (Here $\hat{p}_{i}$ denotes the deletion of the point $p_{i}$.)

Proposition 5.4 $\partial_{n-1} \circ \partial_{n}=0$ for $1 \leq n \leq m .($ See $[9,14])$.

Proof Let $\Delta=\left[x_{0}, \ldots, x_{n}\right]$ be a $(\kappa, n)$-simplex. Then

$$
\begin{aligned}
\partial_{n-1} \circ \partial_{n}(\Delta)= & \partial_{n-1}\left(\partial_{n}\left[x_{0}, \ldots, x_{n}\right]\right) \\
= & \partial_{n-1}\left(\sum_{i=1}^{n}(-1)^{i}\left[x_{0}, \ldots, \hat{x}_{i}, \ldots, x_{n}\right]\right) \\
= & \sum_{i=1}^{n}(-1)^{i}\left[\sum_{j=1}^{n}(-1)^{j}\left[x_{0}, \ldots, \hat{x}_{j}, \ldots, \hat{x}_{i}, \ldots, x_{n}\right]\right] \\
& +\sum_{j=i+1}^{n}(-1)^{j-1}\left[x_{0}, \ldots, \hat{x}_{i}, \ldots, \hat{x}_{j}, \ldots, x_{n}\right] \\
= & \sum_{j<i \leq n}(-1)^{i+j}\left[x_{0}, \ldots, \hat{x}_{j}, \ldots, \hat{x}_{i}, \ldots, x_{n}\right] \\
& +\sum_{i<j \leq n}(-1)^{i+j-1}\left[x_{0}, \ldots, \hat{x}_{i}, \ldots, \hat{x}_{j}, \ldots, x_{n}\right] \\
= & \sum_{i \neq j}\left((-1)^{i+j}+(-1)^{i+j-1}\right)\left[x_{0}, \ldots, \hat{x}_{j}, \ldots, \hat{x}_{i}, \ldots, x_{n}\right]=0 .
\end{aligned}
$$

Definition 5.5 Let $X$ be a digital simplicial group with $\kappa$-adjacency. Then

$$
H_{q}^{\kappa}(X)=\frac{Z_{q}^{\kappa}(X)}{B_{q}^{\kappa}(X)}=\frac{\operatorname{Ker} \partial_{q}}{\operatorname{Im} \partial_{q+1}}
$$

is called the $q$ th digital homology group, where $Z_{q}^{\kappa}(X)=\operatorname{Ker} \partial_{q}$ is the groups of digital simplicial $q$-cycles and $B_{q}^{\kappa}(X)=\operatorname{Im} \partial_{q+1}$ is the groups of digital simplicial $q$-boundaries.

Proposition 5.6 Let $X_{0}=[0]$ be a $(2,0)$-simplex. Then $H_{0}^{2}\left(X_{0}\right) \cong \mathbb{Z}$.

Proof Consider the $X_{0}=[0],(2,0)$-simplex which is shown as

So, $C_{0}^{2}\left(X_{0}\right)=\langle 0\rangle \cong \mathbb{Z}$ ve $C_{i}^{2}\left(X_{0}\right)=\{0\}, i \geq 1$. 
Then we get the short sequence as follows:

$$
0 \stackrel{\partial_{1}}{\longrightarrow} C_{0}^{2}\left(X_{0}\right) \stackrel{\partial_{0}}{\longrightarrow} 0 \text {. }
$$

Since $Z_{0}^{\kappa}\left(X_{0}\right)=\operatorname{Ker} \partial_{0}=C_{0}^{2}\left(X_{0}\right) \cong \mathbb{Z}$, then $B_{0}^{\kappa}\left(X_{0}\right)=\operatorname{Im} \partial_{1}=\{0\}$.

Consequently, $H_{0}^{\kappa}\left(X_{0}\right)=\frac{Z_{0}^{\kappa}\left(X_{0}\right)}{B_{0}^{\kappa}\left(X_{0}\right)}=\frac{\operatorname{Ker} \partial_{0}}{\operatorname{Im} \partial_{1}} \cong \mathbb{Z}$.

Proposition 5.7 Let $X_{1}=[0,1]$ be $(8,1)$-simplex. Then

$$
H_{q}^{\kappa}\left(X_{1}\right)= \begin{cases}\mathbb{Z}, & q=0, \\ 0, & q>0 .\end{cases}
$$

Proof Consider the $X_{1}=[0,1],(8,1)$-simplex as follows.

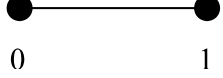

Let us choose the direction as $0<1$. Thus $H_{q}^{8}\left(X_{1}\right) \cong\{0\}$ for $q>1$. Then $C_{0}^{8}\left(X_{1}\right)$ and $C_{1}^{8}\left(X_{1}\right)$ are free abelian groups induced by the following basis, respectively:

$$
\begin{aligned}
& \{\langle 0\rangle,\langle 1\rangle\}, \\
& \{\langle 0,1\rangle\} .
\end{aligned}
$$

Hence we get the following short sequence:

$$
0 \stackrel{\partial_{2}}{\longrightarrow} C_{1}^{8}\left(X_{1}\right) \stackrel{\partial_{1}}{\longrightarrow} C_{0}^{8}\left(X_{1}\right) \stackrel{\partial_{0}}{\longrightarrow} 0 .
$$

We may point out:

$$
\begin{aligned}
& B_{1}^{8}\left(X_{1}\right)=\operatorname{Im} \partial_{2}=\{0\}, \\
& \partial_{1}(a\langle 0,1\rangle)=a(\langle 1\rangle-\langle 0\rangle), \quad a \in \mathbb{Z}, \\
& Z_{1}^{8}\left(X_{1}\right)=\operatorname{Ker} \partial_{1}=a(\langle 1\rangle-\langle 0\rangle)=0 \Rightarrow a=0 .
\end{aligned}
$$

Thus $Z_{1}^{8}\left(X_{1}\right)=\{0\}$. Therefore $H_{1}^{8}\left(X_{0}\right)=\frac{Z_{1}^{8}\left(X_{0}\right)}{B_{1}^{8}\left(X_{0}\right)}=\frac{\operatorname{Ker} \partial_{1}}{\operatorname{Im} \partial_{2}} \cong\{0\}$.

Proposition 5.8 Let $X_{2}=[0,1,2]$ be $(8,2)$-simplex. Then

$$
H_{q}^{8}\left(X_{1}\right)= \begin{cases}\mathbb{Z}, & q=0, \\ 0, & q>0 .\end{cases}
$$

Proof Consider the $X_{2}=[0,1,2],(8,2)$-simplex as follows.

Choosing the direction is $0<1<2$ for this simplex, then $H_{q}^{8}\left(X_{1}\right) \cong\{0\}$ for $q>2$. On the other hand, $C_{0}^{8}\left(X_{2}\right), C_{1}^{8}\left(X_{2}\right)$ and $C_{2}^{8}\left(X_{2}\right)$ are free abelian groups induced by the following 


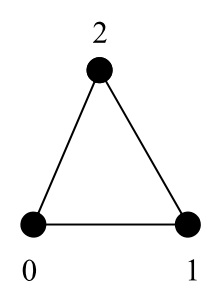

basis, respectively:

$$
\begin{aligned}
& \{\langle 0\rangle,\langle 1\rangle,\langle 2\rangle\}, \\
& \{\langle 0,1\rangle,\langle 1,2\rangle,\langle 0,2\rangle\}, \\
& \{\langle 0,1,2\rangle\} .
\end{aligned}
$$

Hence, we get the following short sequence:

$$
0 \stackrel{\partial_{3}}{\longrightarrow} C_{2}^{8}\left(X_{2}\right) \stackrel{\partial_{2}}{\longrightarrow} C_{1}^{8}\left(X_{2}\right) \stackrel{\partial_{1}}{\longrightarrow} C_{0}^{8}\left(X_{2}\right) \stackrel{\partial_{0}}{\longrightarrow} 0 .
$$

Here $B_{2}^{8}\left(X_{2}\right)=\operatorname{Im} \partial_{3}=\{0\}$.

$$
\partial_{2}(a\langle 0,1,2\rangle)=a(\langle 1,2\rangle-\langle 0,2\rangle+\langle 0,1\rangle) .
$$

So, $Z_{2}^{8}\left(X_{2}\right)=\{0\}$. Thus $H_{2}^{8}\left(X_{2}\right)=\{0\}$.

From the description of $\partial_{2}$, we get

$$
B_{1}^{8}\left(X_{2}\right)=\{a(\langle 0,1\rangle+\langle 1,2\rangle-\langle 0,2\rangle) \mid a \in \mathbb{Z}\}
$$

and

$$
\partial_{1}(a\langle 0,1\rangle+b\langle 1,2\rangle+c\langle 0,2\rangle)=(a-b)\langle 0\rangle+(a-c)\langle 1\rangle+(b+c)\langle 2\rangle=0 .
$$

Therefore $a=-b=c$, so

$$
Z_{1}^{8}\left(X_{2}\right)=\{a(\langle 0,1\rangle+\langle 1,2\rangle-\langle 0,2\rangle) \mid a \in \mathbb{Z}\}=B_{1}^{8}\left(X_{2}\right) .
$$

Thus $H_{1}^{8}\left(X_{2}\right)=\{0\}$. Hence

$$
\begin{aligned}
& \{a\langle 0,1\rangle+b\langle 1,2\rangle+c\langle 0,2\rangle \mid a, b, c \in \mathbb{Z}, a+b+c=0\} \cong \mathbb{Z}^{2}, \\
& a\langle 0,1\rangle+b\langle 1,2\rangle-(a+b)\langle 0,2\rangle=\partial_{1}(-a\langle 0,1\rangle-b\langle 1,2\rangle) .
\end{aligned}
$$

Therefore $B_{0}^{8}\left(X_{2}\right)=\mathbb{Z}^{2}$.

By using a short sequence again, we obtain

$$
Z_{0}^{8}\left(X_{2}\right)=\{a\langle 0\rangle+b\langle 1\rangle+c\langle 2\rangle \mid a, b, c \in \mathbb{Z}, a+b+c=0\} \cong \mathbb{Z}^{3} .
$$

Then $H_{0}^{8}\left(X_{2}\right)=\frac{Z_{0}^{8}\left(X_{2}\right)}{B_{0}^{8}\left(X_{2}\right)}=\mathbb{Z}$. 
Consequently,

$$
H_{q}^{8}\left(X_{2}\right)= \begin{cases}\mathbb{Z}, & q=0, \\ 0, & q>0 .\end{cases}
$$

Theorem 5.9 Let X be a digital simplicial group of dimension 2 with 8-adjacency, then

$$
H_{q}^{8}(X)= \begin{cases}\mathbb{Z}, & q=0 \\ 0, & q>0\end{cases}
$$

Proof Considering Propositions 5.6, 5.7 and 5.8, we have

$$
H_{q}^{8}(X)= \begin{cases}\mathbb{Z}, & q=0 \\ 0, & q>0\end{cases}
$$

\section{Conclusion}

In this paper we introduce the simplicial groups in digital images, and we calculate digital homology group of two-dimensional simplicial group.

\section{Competing interests}

The authors declare that they have no competing interests.

\section{Authors' contributions}

All the authors read and approved the final manuscript.

\section{Acknowledgements}

Dedicated to Professor Hari M Srivastava.

The authors thank the editor and the referees for their valuable suggestions to improve the quality of this paper.

Received: 17 December 2012 Accepted: 8 March 2013 Published: 2 April 2013

\section{References}

1. Rosenfeld, A: Continuous functions on digital pictures. Pattern Recognit. Lett. 4, 177-184 (1986)

2. Han, SE: Computer topology and its applications. Honam Math. J. 25, 153-162 (2003)

3. Kong, TY, A digital fundamental group. Comput. Graph. 13, 159-166 (1989)

4. Kong, TY, Roscoe, AW, Rosenfeld, A: Concepts of digital topology. Topol. Appl. 46, 219-262 (1992)

5. Malgouyres, R: Homotopy in 2-dimensional digital images. Theor. Comput. Sci. 230, 221-233 (2000)

6. Boxer, L: Digitally continuous functions. Pattern Recognit. Lett. 15, 833-839 (1994)

7. Boxer, L: A classical construction for the digital fundamental group. J. Math. Imaging Vis. 10, 51-62 (1999)

8. Boxer, L: Properties of digital homotopy. J. Math. Imaging Vis. 22, 19-26 (2005)

9. Karaca, I, Boxer, L, Öztel, A: Topological invariants in digital images. J. Math. Sci.: Adv. Appl. 11(2), 109-140 (2011)

10. Karaca, I, Boxer, L: Some properties of digital covering spaces. J. Math. Imaging Vis. 37, 17-26 (2010)

11. Karaca, I, Boxer, L: The classification of digital covering spaces. J. Math. Imaging Vis. 32, 23-29 (2008)

12. Kan, DM: A combinatorial definition of homotopy groups. Ann. Math. 61, 288-312 (1958)

13. Freidman, G: An elementary illustrated introduction to simplicial set. Rocky Mt. J. Math. 42, 353-424 (2011)

14. Arslan, H, Karaca, I, Öztel, A: Homology groups of $n$-dimensional digital images. In: XXI. Turkish National Mathematics Symposium, B1-13 (2008)

15. Herman, GT: Oriented surfaces in digital spaces. CVGIP, Graph. Models Image Process. 55, 381-396 (1993)

16. Khalimsky, E: Motion, deformation, and homotopy in finite spaces. In: Proceedings IEEE Intl. Conf. on Systems, Man, and Cybernetics, pp. 227-234 (1987)

17. Munkres, JR: Topology a First Course. Prentice Hall International, Englewood Cliffs (1975)

18. Mutlu, A, Mutlu, B, Öztunç, S: On digital homotopy of digital paths. Res. J. Pure Algebra 2(6), 147-154 (2012)

19. Öztunç, S, Mutlu, A: Categories in digital images. Am. J. Math. Stat. 3(1) 62-66 (2013)

doi:10.1186/1029-242X-2013-143

Cite this article as: Öztunç et al.: The construction of simplicial groups in digital images. Journal of Inequalities and Applications 2013 2013:143. 